\title{
Estado de la investigación en enfermería de América Latina: una revisión de la literatura*
}

\author{
State-of-the-Art of nursing research in Latin America: a literature review \\ Estado da pesquisa em enfermagem na América Latina: revisão da literatura
}

Rusbert Fernando Álvarez del Río

Universidad de Antioquia, Colombia

ORCID: https://orcid.org/0000-0002-7856-6966

DOI: https://doi.org/10.11144/Javeriana.ie23.eiea

Ana Ligia Escobar Tobón ${ }^{\text {a }}$

Universidad de Antioquia, Colombia

ana.escobar@udea.edu.co

ORCID: https://orcid.org/0000-0003-0222-9522

Angela Maria Salazar Maya

Universidad de Antioquia, Colombia

ORCID: https://orcid.org/0000-0001-7599-1193

Recibido: 06 Abril 2021

Aceptado: 07 Septiembre 2021

Publicado: 30 Diciembre 2021

\section{Resumen:}

Introducción: la enfermería requiere analizar la producción de conocimiento para identificar la situación actual de la investigación en América Latina y los desafíos que surgen en el ejercicio profesional. Método: revisión integrativa que contempló la producción científica entre los años 2014 y 2018, según Whittemore, y Whittemore y Knafl, quienes proponen: identificación del problema, búsqueda de literatura, evaluación, análisis, reducción y presentación de resultados. Resultados: emergieron el cuidado de enfermería - humanizado, seguro y culturalmente congruente-, APS y promoción de la salud, cuidado durante el ciclo vital, formación, educación y liderazgo en los servicios de salud y salud en el trabajo. Discusión: el cuidado de enfermería durante todas las etapas del ciclo vital humano requiere la comprensión e investigación de las dimensiones filosófica, disciplinar, heurística, hermenéutica y política que sustenta la práctica científica. Conclusiones: se develan las necesidades por un cuidado humanizado y seguro, que comprenda la diversidad cultural, el fomento de la APS y promoción de la salud, el cuidado durante el ciclo vital, y la búsqueda de un ejercicio digno y seguro, con liderazgo y fortaleza política.

Palabras clave: investigación en enfermería, atención de enfermería, estadios del ciclo de vida, humanización del cuidado, liderazgo.

\section{Abstract:}

Introduction: Nursing needs to analyze the knowledge production in order to identify the state-of-the-art of research in Latin America. Methods: It is a comprehensive review covering the scientific production between 2012 and 2018, after Whittemore, and Whittemore \& Knafl who state: identification of problem, literature search, evaluation, analysis, reduction and result presentation. Results: this review found the following topics: nursing care - humanized, save and culturally congruent-, APS and health promotion, care during the lifetime, formation, education and leadership in healthcare and health at work. Discussion: Nursing care throughout all the stages of the human lifetime needs to understand and research the philosophical, heuristic, disciplinary, hermeneutic and political dimensions that support the scientific practice. Conclusions: it was found that there is a need to count on a humanized and save care, embracing the cultural diversity, the APS format and health promotion, care in the lifetime, and the quest for an appropriate and save practice, with leadership and political strength.

Keywords: nursing research, nursing care, stages of the lifetime, humanization of care, leadership.

\section{Resumo:}

Introdução: a enfermagem requer analisar a produção de conhecimento para identificar a situação atual da pesquisa na América Latina e os desafios que surgem na pratica profissional. Método: revisão integrativa que contemplou a produção científica entre 2014 e 2018, segundo Whittemore, e Whittemore e Knafl, que propõem: identificação do problema, procura de literatura, avaliação, análise, redução e apresentação de resultados. Resultados: surgiram o cuido de enfermagem —humanizado, seguro e culturalmente

Notas de autor

a Autora de correspondencia: ana.escobar@udea.edu.co 
consistente-, APS e promoção da saúde, cuidado durante o ciclo vital, formação, educação e liderança em serviços de saúde e saúde ocupacional. Discussão: o cuidado de enfermagem em todas as etapas do ciclo vital humano requer a compreensão e pesquisa das dimensões AlosóAca, disciplinar, heurística, hermenêutica e política que sustentam a prática cientíAca. Conclusões: desvelaramse as necessidades de um cuidado humanizado e seguro, que compreenda a diversidade cultural, fomento da APS e promoção da saúde, o cuidado durante o ciclo vital e a busca de um exercício digno e seguro, com liderança e força política.

Palavras-chave: pesquisa em enfermagem, atenção em enfermagem, etapas do ciclo de vida, humanização do cuidado, liderança.

\section{Introducción}

Analizar la producción del conocimiento de enfermería en un contexto y tiempo determinado resulta esencial para identiacar temas y perspectivas investigativas, al igual que las necesidades para el desarrollo de la investigación y el mejoramiento de la calidad de vida de personas y colectivos humanos (1).

En las publicaciones de enfermería sobre el rumbo de la investigación en América Latina desde la década de 1990 se identiacaron grandes vacíos en el desarrollo de la teoría, que impidieron la evolución del conocimiento disciplinar (2). En la década 2000-2010, las investigaciones ilustraron una limitada producción cientíaca en enfermería, que se justiacó por la poca formación en investigación que se recibía durante el pregrado.

Posteriormente, en 2017, la Organización Panamericana de la Salud (OPS) y la Organización Mundial de la Salud (OMS) acogieron la estrategia de recursos humanos para el acceso y la cobertura universal de salud, y de esta manera se empezó a vislumbrar un cambio en la formación de los recursos humanos y tecnologías que ayudaran a afrontar las barreras geográAcas, socioculturales, organizacionales y económicas (3).

Aunque en el último quinquenio la inversión en investigación en la región ha aumentado, esta es inversamente proporcional a sus necesidades, además se observa un desconocimiento de la producción cientíaca de enfermería en Latinoamérica.

Esta revisión tuvo como objetivo realizar una aproximación al estado de la investigación en enfermería en América Latina entre los años 2014 y 2018, con el An de facilitar la orientación de futuras indagaciones que aborden los fenómenos de interés de una manera más compleja, y que le permita a la profesión compartir conocimientos, conocer la importancia de lo que ocurre, planiacar investigaciones futuras y fortalecer la práctica.

\section{Material y métodos}

Se realizó una revisión integrativa a la luz de (4) y (5), quienes proponen las siguientes etapas:

\section{Etapa N. 1}

Se identiacó el problema consistente en una carencia de síntesis de información relacionada con el estado de las investigaciones realizadas en América Latina. Surge la pregunta: ¿cuál es el estado de la investigación en enfermería de América Latina entre 2014 y 2018?

\section{Etapa N. 2}

Búsqueda de literatura. Se realizó, entre septiembre y diciembre de 2018, la consulta en las bases de datos Scielo y Lilacs, donde se reúnen artículos de revistas indexadas que han sido sometidos a evaluación. Las palabras clave fueron nursing AND research. enfermería AND investigación. enfermagem 
AND pesquisa. Los criterios de inclusión fueron artículos de investigaciones primarias realizadas por enfermeros latinoamericanos, en texto completo, en español, portugués e inglés, de Centro- y Suramérica, de 2014 a 2018; se excluyeron capítulos de libros, editoriales y tesis. Los artículos repetidos en las bases de datos se consideraron solo una vez. La figura 1 da cuenta del proceso de selección; en total se valoraron 854 artículos.
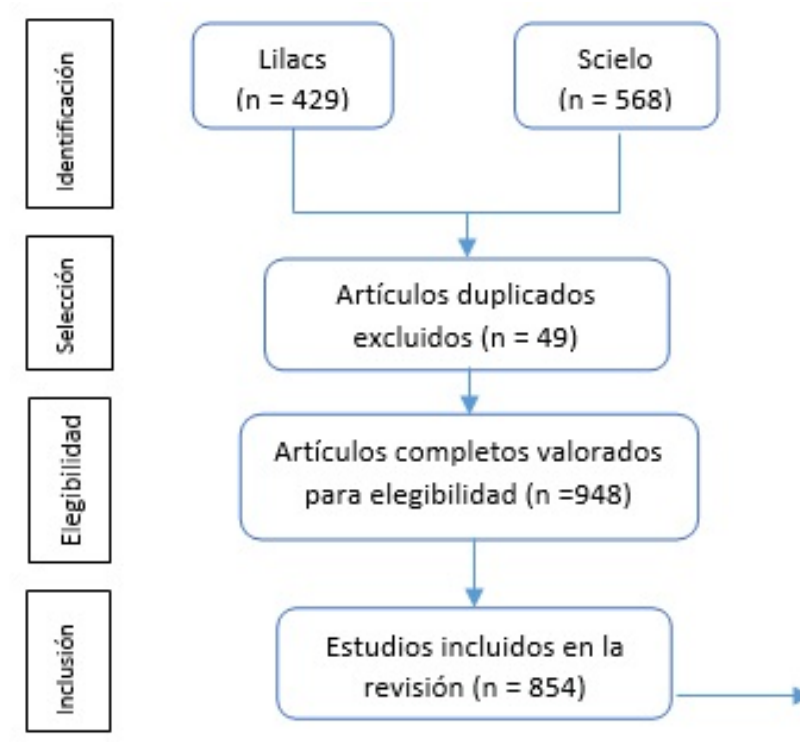

FIGURA 1

Búsqueda de la información

Fuente: elaboración propia.

\section{Etapa N. 3}

Evaluación de datos. Para realizar la interpretación de los artículos se organizaron, codificaron y clasificaron en una base de datos en Excel de acuerdo con los siguientes criterios: título, tema, objetivo, diseño y enfoque de estudio, conclusiones y recomendaciones.

\section{Etapa N. 4}

Análisis y reducción de datos. Según (4), los investigadores leyeron los artículos, y posteriormente los clasificaron por temas; además, se utilizaron herramientas de investigación cualitativa para analizar la información. Los datos se codificaron para la construcción de temas y subtemas relevantes, que dieron respuesta a la pregunta del estudio. Como el objetivo fue ambicioso se encontró una limitación en el estudio, traducida en los 3 años que se requirieron para analizar y clasificar con exhaustividad todos los artículos que cumplieron con los criterios de inclusión.

Los criterios de calidad considerados en el estudio fueron la transferibilidad, que se trabajó enfatizando en el muestreo intencional y sistemático, así como en la descripción de donde se seleccionó cada artículo y periodo estudiado; la credibilidad. auditabilidad, que se trabajó con los pares, quienes revisaron el análisis, en el desarrollo del estudio, que llevaría a la reducción en unidades temáticas, y la confirmabilidad, que se trabajó con la aspiración ética de mostrar transparentemente todo el proceso a través de la triangulación de los datos y de los investigadores; lo anterior permitió visualizar la realidad desde diferentes perspectivas (6). 


\section{Etapa N. 5}

Presentación de resultados. La tabla 1 presenta los temas que emergieron, con sus diseños y enfoques investigativos.

TABLA 1

Síntesis de temas diseños y enfoques de investigación

\begin{tabular}{|c|c|c|}
\hline Tema del artículo & $\begin{array}{l}\text { Diseño de estudio } \\
\text { (número de } \\
\text { investigaciones) }\end{array}$ & $\begin{array}{l}\text { Enfoque metodológico } \\
\text { (número de investigaciones) }\end{array}$ \\
\hline $\begin{array}{l}\text { Cuidado durante el ciclo } \\
\text { vital humano }\end{array}$ & Cualitativo (175) & $\begin{array}{l}\text { + desc (64); integ (36); fenom (21); an-cont } \\
(10) \text {; s-hist (6); an-disc (6); converg (5); t- } \\
\text { fund (5); rep-social (4); herm (3); narrat (2); } \\
\text { etnog (2); inv-acc(2); an-concept (2); an- } \\
\text { represent (2); epi-crítica (1); est-caso(1); a- } \\
\text { crit (1); metasint (1); est-campo (1). }\end{array}$ \\
\hline & Cuantitativo (86) & $\begin{array}{l}+\operatorname{desc}(70) \text {; cas-con (4); exper (3); coh (2); } \\
\text { ecol (2); val-inst (2); rev-sist (1); bibliom (1); } \\
\text { prod-softw (1). }\end{array}$ \\
\hline $\begin{array}{l}\text { Formación, educación y } \\
\text { liderazgo en los servicios } \\
\text { de salud }\end{array}$ & Cuantitativo (67) & $\begin{array}{l}\text { *desc (40); integ (16); s-hist (15); an-cont } \\
(10) \text {; herm (8); est-caso (7); fenom (6); t-fund } \\
\text { (5); etnog (2); an-represent (2); metasint (2); } \\
\text { rep-social (1); an-disc (1); inv-acc (1); epi- } \\
\text { crítica (1). } \\
\text { +desc (49); exper (7); val-inst (3); cas-con (2); } \\
\text { m-anál (2); ecol (2); coh (1); super (1). }\end{array}$ \\
\hline $\begin{array}{l}\text { APS y promoción de la } \\
\text { Salud }\end{array}$ & Cuantitativo (32) & $\begin{array}{l}\text { 'desc (30); integ (9); fenom (8); est-caso (6); } \\
\text { an-concept (4); an-cont (4); herm (4); t-fund } \\
\text { (4); etnog (3); converg (3); est-campo (2); } \\
\text { MCS (2); inv-acc (1); rep-social (1); h-vida } \\
\text { (1). } \\
\text { +desc (22); exper (4); cas-con (4); ecol (1); } \\
\text { val-inst (1). }\end{array}$ \\
\hline $\begin{array}{l}\text { Relevancia de la } \\
\text { investigación }\end{array}$ & Mixto (2) & $\begin{array}{l}\text { ‘integ (16); desc (9); s-hist (5); a-crit (5); s- } \\
\text { hist (4); fenom (3); narrat (2); an-emoc (1); } \\
\text { an-cont (1); t-fund (1); metasínt (1); a-crit (1). } \\
\text { +Val-inst (32); desc (10); bibliom (9); coh } \\
\text { (2); exper (1); cas-con (1); ecol (1). }\end{array}$ \\
\hline $\begin{array}{l}\text { Cuidado de enfermería } \\
\text { humanizado, } \\
\text { culturalmente congruente } \\
\text { y seguro }\end{array}$ & Cuantitativo (44) & $\begin{array}{l}\text { *integ (15); desc (12); an-concep (7); est-caso } \\
\text { (4); t-fund (4); fenom (3); etnog (3); rep- } \\
\text { social (2); a-crit (2); an-cont (1); est-campo } \\
\text { (1); MCS (1); narrat (1); s-hist (1). } \\
\text { + desc (32); coh (5); val-inst (3); cas-con (3); } \\
\text { super (1). }\end{array}$ \\
\hline
\end{tabular}




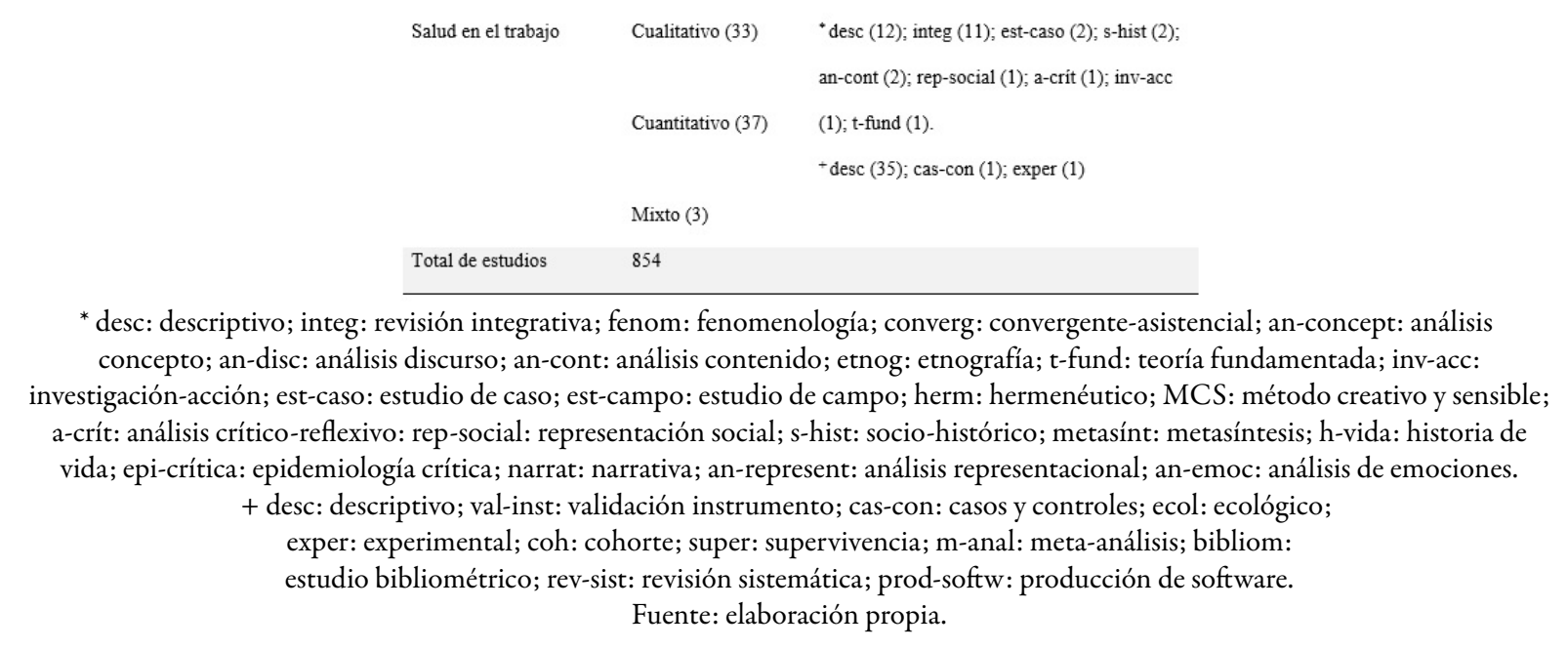

\section{Cuidado de enfermería humanizado, culturalmente congruente y seguro}

Para brindar cuidado humanizado se requiere una formación académica idónea y competente, y una práctica profesional ética. No obstante, dadas las políticas de mercado y contratación laboral, se deben aunar esfuerzos, en todos los escenarios de práctica profesional, que conciban al ser humano en sus particularidades y promuevan las actitudes y comportamientos de no discriminación por identidad, cultura, raza, género u orientación sexual.

La revisión afinca la necesidad de un cambio de valores compartidos entre las personas y la cultura de las organizaciones, para una transformación que contribuya a mejorar la calidad de la atención en salud.

\section{Atención Primaria en Salud (APS) y promoción de la salud}

Ambas estrategias involucran áreas de interés y retos profesionales que se describen de la siguiente manera:

Autocuidado. Es necesario avanzar y proponer la exploración y solución de problemas relacionados con el autocuidado en todos los grupos etarios, con énfasis en poblaciones con edades extremas; se plantea la investigación profesional y el trabajo interdisciplinario para su logro, pensando en las dinámicas poblaciones de cada país de la región y el índice de envejecimiento.

Participación social y cuidado del medioambiente. Es importante valorar las subjetividades de los usuarios, las relaciones que establecen en su dinámica de vida y el apoyo intersectorial, para contribuir con prácticas comprometidas con ambientes y entornos saludables, promoviendo el empoderamiento de la comunidad y la reflexión, en un trabajo por un planeta sostenible.

Educación para la salud. Es imperiosa en la construcción de la autonomía, al considerar que la apropiación de conocimientos actúa como un instrumento de aprendizaje, con el cual el saber científico pasa a formar parte de la vida cotidiana de las personas.

\section{Cuidado durante el ciclo vital humano}

Los hallazgos en este tema plantean:

Atención a la niñez y la adolescencia. Se inclina por garantizar los derechos sociales y de salud de los niños con condición crónica, estableciendo planes de cuidados necesarios y específicos a sus necesidades, con inversión en recursos para la universalidad y la calidad de la atención. Los estudios sugieren adoptar una postura que contribuya a aumentar la autoestima y la capacidad de recuperación en la adolescencia, 
para favorecer la autonomía y protagonismo de sus propias vidas, fomentando estilos de vida saludable, e impactando en su proyecto de vida.

Salud reproductiva, materna y familiar. Se requiere empoderar a las gestantes y sus parejas frente a la salud sexual y reproductiva, y a las prácticas saludables; por tanto, los estudios identiacan los beneAcios del acompañamiento profesional en la aplicabilidad de políticas de anticoncepción.

En lo que se reAere al proceso de hospitalización del binomio madre-hijo, el trabajo apunta a la transición hospital-casa de manera segura y tranquila, moderada por la presencia y la acción de las enfermeras.

Proceso de vejez y envejecimiento. Se centra el interés por la comprensión del fenómeno del envejecimiento y el reconocimiento de los deseos individuales. Este tema se debe investigar en las esferas histórica, social, cultural, espiritual y sexual, además de fortalecer la continuidad de las redes y las instituciones de salud que desarrollan estrategias para el cuidado de enfermería y la prevención del riesgo.

Enfermedad crónica y sobrecarga del cuidador. Es preciso brindar herramientas profesionales desde la gestión, la administración, la promoción de la salud, la prevención de la enfermedad y la educación para la salud, que permitan mejorar las prácticas de las personas, los cuidadores y las familias.

Cuidados al final de la vida. Los estudios revisados invitan a respetar la autonomía de los pacientes en la atención al Anal de la vida y a su vez reclaman nuevos abordajes sobre los cuidados paliativos.

Salud mental. Se encontró una crítica a la función y conceptualización de la enfermería psiquiátrica, con una preocupación sobre la práctica y entrenamiento de enfermería en la institución y en el domicilio. Igualmente se identiacó que el manejo de la drogodependencia exige un enfoque integrado y multidisciplinario.

Violencia en el ciclo vital. Es un problema de salud pública en el mundo y aparece dentro de los grandes desafíos para los investigadores en cuanto al reconocimiento y medición en la población en general. Se destaca el papel fundamental de la academia en la formación profesional y la educación continua con el propósito de contribuir a una atención emancipadora de las víctimas.

\section{Formación, educación y liderazgo en los servicios de salud}

Se aborda en dos ámbitos interrelacionados: el primero es la responsabilidad de la academia durante los procesos de formación; el segundo deane la participación del profesional de enfermería en diferentes escenarios. Se inAere con ello que aprender sobre enfermería es un proceso multidimensional que exige tiempo desde dos perspectivas identiacadas: la primera se relaciona con la supervisión y apoyo de adecuados entornos de aprendizaje, y la segunda, con la práctica de enfermería en el campo, es decir una acción política que debe desarrollarse en los espacios gubernamentales, locales, regionales y globales.

\section{Salud en el trabajo}

Se ilustra la importancia de la prevención de la carga laboral, especialmente en los profesionales de enfermería, y se destaca la necesidad de iniciativas dirigidas a la educación, la reÆexión y la promoción de la salud de los trabajadores. Se comprueba que los factores que diacultan la labor se relacionan con el trabajo en entornos hostiles y bajo cambios climáticos, la exposición al riesgo, la falta de conocimiento o capacitación, y las deAcientes relaciones con el equipo. 


\section{Discusión}

En el contexto latinoamericano, los estudios reflejan la búsqueda continua por fortalecer la disciplina mediante la investigación, la cual permite la proyección profesional, sobrepasa las rutinas de los procedimientos y facilita la sistematización del conocimiento con responsabilidad ética y compromiso político (7), con el fin de contribuir al mejoramiento de las condiciones ideales de vida, en general, y de salud, en particular, con mejores servicios que se ofrecen según las necesidades de salud de la población.

El cuidado de enfermería durante el ciclo vital permanece en constante transición entre la asistencia hospitalaria y el enfoque comunitario; actúa en la atención al niño $(8,9)$ y al adolescente (10-12), a la familia gestante (13-15), al adulto joven (16,17) y al adulto mayor (18); además, invita a la reflexión, madurando en sus procesos de salud-enfermedad y sus diferentes dimensiones con especialidad, cientificidad y humanidad en la intervención de enfermedades crónicas y degenerativas (19), enfermedades catastróficas (20), formas de violencia $(21,22)$ y relación con el medioambiente $(23)$; por tanto, se requiere responder con efectividad para el logro de la calidad de vida y salud.

En cuanto a la APS y la promoción de la salud, (24) las fundamentan en el cuidado familiar para la búsqueda de desarrollo y bienestar; sin embargo lo encontrado en la revisión frente a la APS (25-27) se relaciona con la existencia aún de problemas estructurales de servicios de salud, entre ellos el acceso y la disponibilidad de los profesionales, la desintegración de los sistemas de salud y la limitación del poder para solucionar problemas por parte de los equipos primarios, situaciones que hacen necesario trabajar en el empoderamiento de las familias y comunidades para las prácticas promotoras de salud.

El cuidado de enfermería humanizado, culturalmente congruente y seguro, es una preocupación para los profesionales y una prioridad interdisciplinaria; como eje transversal de la acción profesional asistencial y comunitaria. (28) nos lleva a reflexionar sobre los atributos indispensables en la realización del cuidado humanizado: el ser humano, la relación profesional-usuario, el sujeto del cuidado, y la comunicación y enfoque holístico. Afirma este autor que una práctica sin la comprensión de las propiedades inherentes al cuidado solo lleva a la realización de la atención, mediante una relación vertical-unidireccional entre el profesional y el usuario.

La formación, educación y liderazgo en los servicios de salud son aspectos que permiten la realización y proyección del profesional de enfermería para responder adecuadamente a los desafíos de los contextos (29). Las escuelas de formación de enfermería en América Latina proyectan un profesional con habilidades para la vida, para el trabajo intraprofesional e interprofesional, que respeta y comprende los procesos inherentes a la vida, la individualidad, la comunidad y la cultura.

Por último, la salud en el trabajo es una preocupación para las agremiaciones y los profesionales (30), en tanto se requiere un esfuerzo continuo por disminuir la sobrecarga laboral en todos los escenarios de desempeño, las deficientes condiciones de contratación y las políticas institucionales que denigran el ejercicio autónomo y liberal. En palabras de (31, 32 y 33), se requiere estimular a los profesionales en la generación de evidencia científica en el área de enfermería en salud ocupacional que mejore las condiciones de trabajo que puedan influir en la calidad del cuidado que otorga y en su propia salud.

\section{Conclusión}

La investigación en enfermería en América Latina ilustra la preocupación por complementar los métodos y ayudar a una mejor comprensión de los fenómenos de cuidado. Asimismo, devela las inquietudes por un cuidado de enfermería humanizado y seguro que comprenda la diversidad cultural, el fomento de la APS y la promoción de la salud, el cuidado durante el ciclo vital y la búsqueda de un ejercicio digno y seguro, con liderazgo y fortaleza política. 


\section{Referencias}

1. Hopia H, Latvala E, Liimatainen L. Reviewing the methodology of an integrative review. Scand J Caring Sci. 2016;30(4):662-69. doi: https://doi.org/10.1111/scs.12327

2. Ailinger RL, Najera RM, Castrillón MC, Manfredi M. Nursing research in Latin America: 1988-1998. Rev Lat Am Enfermagem. 2005;13(6):925-28. https://doi.org/10.1590/S0104-11692005000600002

3. Organización Panamericana de la Salud, Organización Mundial de la Salud. Estrategia de recursos humanos para el acceso universal a la salud y la cobertura universal de salud [Documento PDF]. Washington DC: OPS; 2017. [citado 2021,febrero,26] Disponible en https://iris.paho.org/bitstream/handle/10665.2/34198/CE160-18-s.p df?sequence $=2 \&$ isAllowed $=y$

4. Whittemore R. Combining evidence in nursing research: methods and implications. Nurs Res 2005;54(1):56-62. DOI: https://doi.org/10.1097/00006199-200501000-00008

5. Whittemore R, Knafl K. The integrative review: updated methodology. J Adv Nurs 2005;52(5):546-53 DOI: htt ps://doi.org/10.1111/j.1365-2648.2005.03621.x

6. Cypress BS. Rigor or reliability and validity in qualitative research: perspectives, strategies, reconceptualization, and recommendations. Dimens Crit Care Nurs 2017;36(4):253-63.doi: https://doi.org/10.1097/DCC.00000000 00000253

7. Benítez J. La importancia de la investigación en Enfermería. Enferm Investiga. 2020;5(1):1-2. Disponible en https: //revistas.uta.edu.ec/erevista/index.php/enfi/article/view/826

8. Escobar-Tobón AL. La fiebre en el niño: una mirada reflexiva a las prácticas de cuidado. Av Enferm. 2017;35(3):333-44. https://doi.org/10.15446/av.enferm.v35n3.54848

9. Tavares TS, Duarte ED, de Sena RR. Social rights of children with chronic conditions: a critical analysis of Brazilian public policies. Esc Anna Nery. 2017;21(4). https://doi.org/10.1590/2177-9465-ean-2016-0382

10. Netto de Brum C, Cardoso de Paula C, de Mello Padoin SM, Spiegelberg Zuge S. Experience of diagnosis disclosure for teenagers with HIV. Texto Contexto Enferm. 2016;25(4):1-6. https://doi.org/10.1590/0104-0707201600 1760015

11. Pinheiro Beserra E, Barbosa Sousa L, Peres Cardoso V, Santos Alves MD. Percepção de adolescentes acerca da atividade de vida "exprimir sexualidade". J Res: Fundam Care. 2017;9(2):340-46. https://doi.org/10.9789/217 5-5361.2017.v9i2.340-346

12. Nunes-Queiroz AAFL, Lopes de Sousa AF, de Moura-Feitosa JJ, Sampaio-Nery I, Batista Moura ME. Sex education for adolescents by teachers from a community education center. R Pesq Cuid Fundam. 2016;8(4):5120-125. DOI: https://doi.org/10.9789/2175-5361.2016.v8i4.5120-5125

13. Naidon ÂM, Tatsch-Neves E, da Silveira A, Felix-Ribeiro C. Gestação, parto, nascimento e internação de recémnascidos em terapia intensiva neonatal: relato de mães. Texto Contexto Enferm. 2018;27(2):1-9. https://doi.or g/10.1590/0104-070720180005750016

14. Vasconselos-Amorim T, de Oliveira-Souza Í, de Oliveira-Salimena AM, Azevedo-Queiroz AB, Vasconcelos-Moura MA, Cardoso de Melo MCS. Risco reprodutivo em gestantes portadoras de cardiopatia: o mundo vivido direcionando o cuidado em saúde. Texto Contexto Enferm. 2018;27(2):2-9. https://doi.org/10.1590/0104-07 0720180003860016

15. Demarche-Frutuoso L, Brüggemann OM, Monticelli M, de Oliveira ME, Costa R. Percepções do acompanhante de escolha da mulher acerca da organização e ambiência do centro obstétrico.J Res Fundam Care. 2017;9(2):363-70. DOI: https://doi.org/10.9789/2175-5361.2017.v9i2.363-370

16. Guerrero-Núñez $S$, Valenzuela-Suazo $S$, Cid-Henríquez P. Effective universal coverage of diabetes Mellitus type 2 in Chile. Rev Latino-Am Enferm. 2017;25:1-8. DOI: http://dx.doi.org/10.1590/1518-8345.1630.2871

17. Rivas-Riveros E, Alarcón-Pincheira M, Gatica-Cartes V, Neupayante-Leiva K, Schneider-Valenzuela MB. Escalas de valoración de dolor en pacientes críticos no comunicativos: revisión sistemática. Enferm. 2018;7(1):115-29. https://doi.org/10.22235/ech.v7i1.1544 
18. Machado-Pinheiro F, Santo FHE, de Pinho-Chibante CL, Cardoso-Pestana L. Peral de idosos hospitalizados segundo Viginia Henderson: contribuições para o cuidado em enfermagem. Cuid Fund. 2016;8(3):4789-795. DOI: http://dx.doi.org/10.9789/2175-5361.2016.v8i3.4789-4795

19. Ramírez-Perdomo CA, Solano-Ruiz MC. Social construction of the experience of living with chronic kidney disease. Rev Lat Am Enferm. 2018;26:e3028. DOI: https://doi.org/10.1590/1518-8345.2439.3028

20. Lopes M, Nascimento LC, Zago MMF. Paradox of life among survivors of bladder cancer and treatments. Rev Esc Enferm USP. 2016;50(2):222-29. https://doi.org/10.1590/S0080-623420160000200007

21. Biazus-Dalcin C, Stein-Backes D, Batistin-Zanatta F, Macedo de Sousa FG, Heckler de Siqueira HC, Netto de Oliveira AM. Factors associated with violence in schools: extending knowledges and practices for nursing. Texto Contexto Enferm. 2016;25(4):1-10. http://dx.doi.org/10.1590/0104-07072016004530014

22. de Albuquerque-Netto L, Vasconcelos-Moura MA, França-Araujo CL, do Nascimento-Souza MH, Fernandes e Silva G. Social support networks for women in situations of violence by an intimate partner. Texto Contexto Enferm. 2017;26(2):1-11. https://doi.org/10.1590/0104-07072017007120015

23. Ferreira-Barreto B, Pires-Silva R, Camacho A, Oliveira BGR, Cavalcanti Valente GS. Áe interference of stress on worker nursing in hospital environment and its relation as a risk of factor for the occurrence of câncer. Esq Cuid Fundam. 2016;8(2):4154-167. DOI: https://doi.org/10.9789/2175-5361.2016.v8i2.4154-4167

24. Olaya-Cuadros BL, Gaviria-Noreña DL. Cuidado de la salud de la familia. Rev Cienc Cuidad. 2017;14(1):79-94. DOI: https://doi.org/10.22463/17949831.808

25. Costa-Moreira D, Bispo-Júnior JP. Experiencias y desafíos para la inclusión profesional en la atención primaria de salud en Brasil. Rev Cub Salud Pública. 2020;46(2):1-18. Disponible en https://scielosp.org/article/rcsp/2020 .v46n2/e1569/es/

26. Velásquez VF, Barreto Y, López L. Empoderamiento de líderes comunitarias afrocolombianas desde la Atención Primaria de Salud. Av Enferm. 2017;35(2):133-47. https://doi.org/10.15446/av.enferm.v35n2.54986

27. Vera-Rodríguez JE, Rojo-Pérez N, Quiñones-Varela IS. Sistema de acciones socio sanitarias de enfermería comunitaria implementada en un asentamiento rural cubano. Rev Hum Med. 2016;16(1):130-43. Disponible en: http://scielo.sld.cu/scielo.php?script=sci_arttext\&pid=S1727-81202016000100009\&lng=es\&nrm=iso

28. Cruz Riveros C. La naturaleza del cuidado humanizado. Enfermería: Cuidados Humanizados. 2020;9(1):21-32. h ttp://dx.doi.org/10.22235/ech.v9i1.2146

29. Benavides-Couto A. Liderazgo en enfermería, una necesidad actual. Medisur. 2017;15(2)178-79. Disponible en: $h$ ttp://scielo.sld.cu/scielo.php?script=sci_arttext\&pid=S1727-897X2017000200005\&lng=es\&nrm=iso

30. Zapata-Herrera M, Zapata-Gómez NE. Condiciones de trabajo de las enfermeras y las formas de contratación en Medellín, Colombia. Rev Univ Salud. 2015;17(2):212-23. Disponible en http://www.scielo.org.co/scielo.php? script $=$ sci_arttext\&pid=S0124-71072015000200007

31. Escobar-Tobón AL, Arias-Valencia MM, Salazar-Maya AM. Angustia en cuidadores de niños con Aebre: análisis del concepto. Modelo híbrido. Rev Cienc Cuidad [Internet]. 2018;15(2):65-78. https://doi.org/10.22463/17 949831.1402

32. Reyes-Luna JD, Valenzuela-Suazo S, Rodríguez-Campo V. Enfermería en salud ocupacional: una mirada a los instrumentos utilizados en investigación a nivel internacional. Enferm Actual Costa Rica. 2019;37:188-205. Disponible en https://www.scielo.sa.cr/scielo.php?script=sci_abstract\&pid=S1409-45682019000200188\&ln $\mathrm{g}=\mathrm{es}$

33. Luengo Martínez C, Sanhueza O. Condiciones de trabajo y su relación con la calidad del cuidado y salud del profesional de enfermería. Med Segur Trab. 2016;62(245):368-80. Disponible en http://scielo.isciii.es/scielo.p hp?script $=$ sci_arttext\&pid=S0465-546X2016000500008\&lng=es

\section{Notas}

* $\quad$ Artículo de revisión integrativa 
Licencia Creative Commons CC BY 4.0

Financiamiento: Universidad de Antioquia.

Conflicto de intereses: Los autores declaran no tener con Aicto de intereses.

Cómo citar este artículo: Escobar Tobón A. L., Álvarez del Río R. F., Salazar Maya A. M. Estado de la investigación en enfermería de América Latina: una revisión de la literatura. Investigación en Enfermería, Imagen y Desarrollo. 2021;23. https://doi.org/10.11144/Javeriana.ie23.eiea 